\title{
edoc
}

Institutional Repository of the University of Basel

University Library

Schoenbeinstrasse 18-20

CH-4056 Basel, Switzerland

http://edoc.unibas.ch/

Year: 2009

\section{Bioreactor-based roadmap for the translation of tissue engineering strategies into clinical products}

\author{
Martin, Ivan and Smith, Timothy and Wendt, David
}

Posted at edoc, University of Basel

Official URL: http://edoc.unibas.ch/dok/A6004607

Originally published as:

Martin, Ivan and Smith, Timothy and Wendt, David. (2009) Bioreactor-based roadmap for the translation of tissue engineering strategies into clinical products. Trends in biotechnology, Vol. 27. S. 495-502. 


\title{
BIOREACTOR-BASED ROADMAP FOR THE TRANSLATION OF TISSUE ENGINEERING STRATEGIES INTO CLINICAL PRODUCTS
}

\author{
Ivan Martin $^{1}$, Timothy Smith ${ }^{2}$, David Wendt ${ }^{1}$
}

${ }^{1}$ Departments of Surgery and of Biomedicine, University Hospital Basel, Hebelstrasse 20, 4031 Basel, Switzerland

${ }^{2}$ Octane Medical Group, 640 Cataraqui Woods Drive, Kingston, Ontario, K7P 2Y5 Canada

Corresponding author: I. Martin (imartin@uhbs.ch) 


\section{ABSTRACT}

Despite the compelling clinical need to regenerate damaged tissues/organs, the impressive advances in the field of tissue engineering have yet to result in viable engineered tissue products with wide-spread therapeutic adoption. Although bioreactor systems have been proposed as a key enabling factor in the manufacture of standardized and cost-effective engineered products, this concept appears slow to be embraced and implemented. Here we address scientific, regulatory and commercial challenges intrinsic to the bioreactor-based translation of tissue engineering models into clinical products, proposing a roadmap for the implementation of a new paradigm. The roadmap underlines that bioreactors must be implemented throughout product development, allowing the scientific, medical, industrial and regulatory parties to address basic research questions, conduct sound pre-clinical studies, and ultimately facilitating effective commercialization of engineered clinical products. 


\section{INTRODUCTION}

In the last couple of decades, the appealing possibility of combining living cells with suitable carriers for the regeneration of damaged or lost tissues and organs has promoted the interaction of scientists, engineers, clinicians and business people, leading to the establishment and progressive consolidation of the field of tissue engineering. Examples of successful clinical implementation of the developed concepts include restoration of corneal surfaces [1,2], replacement of a bronchus segment [3], reconstruction of bone [4] and cartilage defects [5], as well as of diseased bladder [6]. Despite significant achievements and enormous clinical demand, the clear need for viable tissue engineered products that are broadly available to patients as part of the routine toolkit of medical treatments still remains unsatisfied. On the one hand, this may be due to a relatively limited establishment of prospective, randomized clinical studies, demonstrating reproducibly superior effectiveness of engineered grafts as compared to conventional treatments. On the other hand, addressing manufacturing-related issues has been proposed to be key for the success of cell-based engineered products [7]. Indeed, as in other applications of biotechnology (e.g., for the production of antibodies or molecular vaccines), successful clinical use of engineered tissue products, as well as their commercial exploitation, may be critically dependent on the introduction of bioreactor-based manufacturing systems [8]. Bioreactors, intended as a means to generate and maintain a controlled physico-chemical culture environment, indeed represent a key element for the automated, standardized, traceable, cost-effective, safe and regulatory compliant manufacturing of cell-based products or engineered grafts for clinical applications [9,10] (Figure 1). However, notwithstanding the promise of a few pioneering systems currently under development or clinical testing (e.g., Octane Biotech Inc., Canada http://www.octaneco.com/biotech and Aastrom Biosciences Inc., USA http://www.aastrom.com) this concept has not yet broadly facilitated the transfer of cell-based processes into clinically and commercially viable therapeutic solutions. In this paper, we 
address the scientific, regulatory and commercial challenges that are hampering the bioreactor-based translation of tissue engineering into clinical products, and based on these issues, propose a roadmap for the implementation of a new paradigm. The new paradigm is fundamentally rooted in the perspective that sensor-based bioreactors must be deployed and validated throughout the product development pipeline, from initial conception until manufacturing of the implant.

\section{SCIENTIFIC ASPECTS}

The introduction of bioreactors in the field of tissue engineering was initially advocated as a powerful means to apply defined regimes of physical forces, with the ultimate goal to regulate and possibly improve the mechanical functionality of the resulting engineered grafts [11]. This approach has advanced scientific understanding of mechanoregulation of developing tissues and emphasized the importance of controlled physical conditioning in tissue regeneration. However, the effective need to apply mechanical forces in vitro to generate more functional grafts is still controversial. Indeed, beyond a few cases where graft functionality might need to be fully developed prior to implantation, such as for engineered blood vessels [12] or heart valves [13], it is becoming increasingly clear that tissue maturation could be more efficiently induced by the physiological biochemical and mechanical cues of our body as an 'in vivo bioreactor' [14], provided that an appropriate quality "starting material” is implanted. Moreover, introducing a mechanical loading regime that correctly emulates the dynamic physiology of the body in order to generate a fully functional tissue would likely make the manufacturing process too complicated and lengthy, and as a result, too expensive and impractical.

In principle, as compared to smart functionalized materials or drug delivery devices, cellbased grafts have the potential to provide superior clinical outcomes, due to the multivalent biological activity of cells (e.g., multiple growth factor release, self-contribution to tissue 
regeneration) and/or of the deposited extracellular matrix (e.g., efficient storage and release of morphogens, physiologically functional mechanical properties). However, engineered products will only be a viable and competitive option against the upcoming off-the-shelf block busters in regenerative medicine [7] if they are manufactured with reproducible properties, a prerequisite for consistent clinical outcomes. This important target is mainly challenged by the intrinsic variability, often not sufficiently underlined, in the behavior of human cells from different batches or donors [15], as well as by the sensitivity of cells to perturbations in the culture environment. While the spotlight of bioreactor-based tissue engineering has typically been on enhancing the functionality or maturation stage of the resulting engineered tissues, the potential for controlled sensor-based bioreactor systems to minimize process and product variability should receive equal attention. By monitoring and controlling physico-chemical culture parameters, bioreactors can help to standardize both the required bioprocesses and the resulting engineered graft, and ensure the automated protocols are compatible with regulatory and commercialization requirements (see sections below and Text Box 1). Moreover, by streamlining culture processes and bypassing operator/handlingdependent procedures, bioreactor-based systems have the potential to increase the robustness and stability of the graft manufacturing process [16,17].

Clearly, in order to broadly deliver on the described opportunities, continued progress against dominant challenges remains essential. These are not only related to the limited availability of on-line, non-invasive 'sensing' techniques for important culture parameters (e.g., cell number, differentiation stage, metabolic activity), but also to the limited fundamental understanding of the cellular and molecular processes underlying regeneration and function of specific tissues and organs. Indeed, to better control and standardize key output product characteristics such as cell identity, quality, purity and potency, the mode of action associated with tissue regeneration should be identified and validated. For example, in most cases it is not yet established whether the performance of a specific engineered product is dependent upon the 
number of implanted cells, the cell phenotype at the time of implantation, the amount of specific extracellular matrix proteins deposited, or the profile of released cytokines. Understanding these scientific uncertainties will help to identify relevant biomarkers, including extracellular matrix components, proteins indicative of proliferative/differentiation stage and metabolites, which can serve as critical process quality control points and predict potency of a tissue engineered product [18]. Based on this necessary fundamental knowledge, the next challenge will then be to define which ranges of specific biochemical, metabolic, and/or environmental culture parameters (e.g., growth factors, glucose, $\mathrm{pH}$, etc.) are required to guarantee reproducible potency features. In this regard, it is clear that the monitoring and control capabilities provided by bioreactor systems, linked to the scientific knowledge obtained through the application of defined and controlled sequences, provides a valuable feedback loop to refine the culture processes and optimize the properties of the engineered product.

\section{REGULATORY ASPECTS}

The clinical introduction of engineered tissue products will likely involve significant regulatory oversight, with an additional degree of complexity as compared to policies and guidance recently implemented on different international levels for cell therapy [19-21]. While manufacturing strategies based on conventional manual cell culture techniques might find difficulties in complying with the new regulatory framework, bioreactor-based manufacturing processes inherently involve automation and reproducibility that facilitates compliance with regulatory objectives. In particular, monitoring process parameters and specific properties of the developing/final graft will allow for a higher level of traceability of key manufacturing data related to identity, purity, and potency of the implant. Moreover, by minimizing the required manual procedures of handling the cells and tissue constructs by the operator, automated bioreactor systems with control platforms and software-based data 
management will facilitate compliance to safety regulations. Ultimately, a stand-alone, fullyautomated and closed system would provide a GMP-compatible manufacturing unit complete with environment control, full operational traceability and failsafe protection measures that go well beyond simplistic automated cell culture. In essence, this is in itself a kind of "GMP-ina-box" concept, with the potential to reduce dependence on large, costly and sometimes not easily available regulatory-compatible facilities (see Text Box 1).

In order for these opportunities to be translated into effective ways to facilitate a more widespread use of tissue engineered products in the clinic, we foresee commonly underestimated difficulties and challenges, related to the practical details of implementation. First, the often rather undefined mode of action of engineered tissues and the limited availability of potency markers on a tissue level eclipses pragmatic testing protocols that can be realistically deployed in the production process (see section on scientific aspects). Issues related to the lack of certainty with which the product output provides the expected patient benefit challenges a credible plan for 'validation', namely the ability to predict performance with a high degree of confidence in the absence of direct data. The validation of production processes is pivotal to regulatory compliance and needs considerable attention in preparation for regulatory review.

The clinical deployment of bioreactors for the preparation of engineered tissues inherently depends upon the safe, reliable and user friendly operation of the system in production environments. Fundamental to any regulatory assessment is the comprehensive analysis of product, process, and environment risk, along with the specific implementation of risk mitigation practices to address 'critical' risks. Risk criticality is assessed based on occurrence, severity and detection, with all factors playing a crucial role in identifying key nodes for active risk management. As for other fields of biotechnology, the use of standardized and automated systems (in this case bioreactor-based manufacturing systems) enables critical risk factors associated with operator handling and the production process to be 
successfully mitigated. However, an overview of risk and risk control associated with the use of bioreactor-systems for the production of engineered tissues, illustrated in Table 1, indicates the complexity and large spectrum of processes involved. Indeed, skepticism by regulatory authorities on the implementation of bioreactor-based graft manufacturing could be tempered by introducing a sound risk assessment early enough in product development.

Beyond the characterization and mitigation of risk associated with the automated tissue engineering process, the regulatory approval of bioreactor systems for production of cellbased implants for clinical use will be highly dependent upon the compliance of the surrounding production environment. For facilities with pre-approval for cell manipulations, the integration of additional equipment to further the culturing of the implant would be significantly more straightforward (although appropriately challenging on the details of the process) than implementation in the more open environment of a specialized clinic. In this regard, uncertainties on the interpretation of 'closed system', especially considering the necessary sampling for off-line monitoring (e.g., for sterility tests) or the need for direct intervention based on "out of specification" (OOS) signals, make it difficult to identify precise rules on the practicality, from a regulatory/safety standpoint, of the "GMP-in-a-box" concept.

\section{COMMERCIAL ASPECTS}

While the promise of tissue engineering has captivated many enthusiasts and has generated significant international investments in research, commercially engineered products are confronted with tough economic realities related to uncertain cost-benefit performance and ultimate eligibility for reimbursement. The limited and perhaps discouraging commercial progress to date could be related to the fact that the basic procedures for generating engineered tissues have generally been based around conventional manual bench-top cell and tissue culture techniques. These manual techniques seem to be particularly appealing during 
initial stages of product development (particularly for start-up initiatives) since the simple and wide-spread manual approach is generally viewed as being a route to minimize initial development time and investment costs, and allow for quick entry into clinical trials and to the market. However, down the line as scientific, technical and commercial momentum builds, production costs associated with manual production quickly become a barrier, in addition to the fact that the traditional techniques possess inherent risks for contamination and intra-/inter-operator variability. Furthermore, pragmatic limits on the escalation of unit production volumes (up-scaling) and related inefficiencies in traceability result in significant challenges to business models that had been founded on manual protocols. As an alternative, a closed, standardized, and operator-independent bioreactor-based production system would possess great benefits in terms of safety and regulatory compliance, and despite incurring high product development costs initially, would have great potential to improve the costeffectiveness of a manufacturing process in the long run, while maximizing the potential for process scale-up (see Text Box 1).

The advantages of a bioreactor-based approach appear convincing and yet bioreactor technology is not widely adopted. This cannot be due to a lack of competent bioreactor designs, as one only needs to perform a literature or patent search to appreciate the plethora of various bioreactors developed to date. So why have we not seen more of a drive to implement bioreactors for the manufacturing of engineered products for clinical applications?

Perhaps the clinical vision in each of the different segments of tissue engineering is still relatively immature and hence quality, validation and productivity demands are considered secondary challenges. One contributing factor to the delayed introduction of novel bioreactor technology could be the required change of mindset to break from well-established cell culture methods. To make this leap, and for bioreactors to gain greater general acceptance, simple and more user-friendly bioreactor systems will have to be introduced during the research phase, given that the routine handling of complex and technically challenging 
systems may discourage their introduction into laboratories that lack a specialized and trained work force. Moreover, even if one does acknowledge that bioreactors can generate engineered tissues of higher quality, there is currently little data highlighting the process reproducibility and - to the best of our knowledge - no sound analysis that critically assesses the potential cost-effectiveness of a bioreactor-based production system. Indeed, economic tools to help guide investment decisions and to evaluate the potential cost-effectiveness of an engineered product are complex, rely on strong assumptions, and are not yet well-established. As a first step, typical methods for the analysis of factors influential in strategic planning need to be applied to the commercialization of bioreactor-based tissue engineering for the clinic. Without reference to a specific clinical indication, the results from a SWOT (Strengths, Weaknesses, Opportunities and Threats) analysis (see Table 2) indicate that the adoption of bioreactors in the clinical delivery of tissue engineering could indeed introduce attractive strengths and opportunities. Not surprisingly, the new bioreactor-based approach also carries certain weaknesses and invokes threats that need to be addressed as part of a well developed clinical, regulatory and reimbursement plan. In order to derive more quantitative indications, the use of a Headroom Method has been proposed as a simple but rigorous way to make preliminary conclusions as to the cost-effectiveness of a new tissue engineering treatment [22]. Difficulties with the implementation of such analytical tools are related to the fact that some critical parameters required as input, for example the expected improvement of clinical performance, can only be arbitrarily assumed, in lieu of the uncertainties associated with an undeveloped technology.

The lack of cost-benefit analyses in the context of clinical efficacy precludes a plausible assessment of the likelihood of reimbursement coverage for the production and surgical implantation of engineered products, which in turn severely impacts the credibility of commercial business plans founded on the widespread use of tissue engineering. Taken together, challenges to the tissue engineering business model coupled with budding but as yet 
not robust demand for bioreactor systems, it is not surprising that industry has been hesitant to embrace commercialization of engineered products and invest significant resources upfront to develop and/or utilize sophisticated manufacturing technology.

Closer interactions between scientists/engineers and the other involved stakeholders (e.g., regulatory bodies, clinicians, industrial partners, health care providers) must therefore aim at an integrated bioreactor design, deployed in a system which can be practical and economical for clinical use. We now have nearly two decades of bioreactor technology for engineered tissues at our disposal, and although these research-oriented systems are generally too complex, user-unfriendly, unsafe, and expensive to be directly utilized for clinical applications, their underlying principles could nevertheless lay a solid foundation for more clinically compliant manufacturing systems. This will require the identification of only the most essential processes, culture parameters, and construct parameters that must be monitored and controlled in order to standardize production and provide meaningful quality and traceability data, but which minimizes risks, costs, and user-complexity.

In this regard, it is questionable whether complex and rather costly automated/robotic systems, which essentially mimic established manual procedures, could actually demonstrate a real cost-benefit by replacing manual cell culture techniques in a manufacturing process. Moreover, current automation techniques may fail to capture the expert nature and role of the cell culture technician. Instead, bioreactor designs could be dramatically simplified, and related development costs significantly reduced, if we re-evaluate the conventional tissue engineering paradigms and implement novel concepts and techniques that could streamline the numerous individual bioprocessing steps. Simplified tissue engineering processes could be key to future manufacturing strategies by requiring only a minimal number of bioprocesses and unit operations, thereby facilitating simplified and compact bioreactor designs with limited automation requirements, with the likely result of reduced product development and operating costs. Here we underline the importance of simplicity and minimal costs while 
maintaining core attributes of implant consistency and overall manufacturing productivity and scalability. And while we have attempted to draw several parallels to bioreactors in other fields of biotechnology, the analogy begins to break down during up-scaling, especially in the context of autologous implants. Given that cells from each patient will be highly variable and must be processed as completely independent batches, we cannot simply upscale the total volume as we would a fermentation process or even for an allogeneic product. Alternatively, the clinical need requires "scaling out" (i.e., to replicate the same bioprocesses at multiple sites) simply by adding additional (low-cost) units to the production as product demand increases [23] (see Figure 1).

Finally, we must ask where the potential exploitation of bioreactors lies within the broad spectrum of tissue engineering applications. Could there be a market for a simple and userfriendly bioreactor for general use in basic research applications? Are hospitals a viable market? A closed "GMP-in-a-box" system would allow competent hospitals to manufacture engineered grafts on-site, without reliance upon a centralized industrial manufacturing facility. On the other hand, would the predominant market lie in industrial firms producing engineered products in-house, with the perspective of introducing automated bioreactors within their manufacturing process? At the present stage, none of these markets can be excluded, and it is likely that business models will have to be adapted to some specific upfront decisions. For example it is possible that a centralized manufacturing facility is appropriate for the engineering of allogeneic grafts, whereas hospital-based production could become a reality for autologous cell-based tissues.

\section{CONCLUSIONS}

The use of bioreactor-based platforms for the translation of tissue engineering strategies into clinical products offers attractive opportunities for exploitation, as well as the potential for broad implementation of cell-based grafts as therapeutic solutions. However, in order to make 
this paradigm successful, several challenges of diverse nature need to be realistically considered and constructively addressed, as outlined in the roadmap proposed in Figure 2. The roadmap advocates that bioreactor-based manufacturing concepts need to be introduced in the development of a tissue engineered product as early as possible. This will allow academics, developers, and industry participants to (i) properly address underlying research questions (e.g., offering the possibility to test the effect of controlled changes in discrete parameters), (ii) carry out sound pre-clinical studies (e.g., offering the possibility to test the in vivo performance of grafts with reproducible features), and (iii) maintain strong ties with regulatory and commercial dimensions (e.g., offering the possibility to implement scaling concepts and compliance with safety requirements). The proposed roadmap also conveys that scientific, regulatory and commercial aspects should all be considered in each of the translational stages, from the initial conception to the final implementation of a clinical product, leading to continuous refinements and beneficial corrections.

Last but not least, success of bioreactor-based tissue engineering products will also depend on the acceptance of the proposed paradigms by the involved stakeholders (e.g., surgeons, industry alliance partners [24], investors, health insurance companies), as well as by the general public (i.e., the potential patient population) [25]. In fact, while the availability of therapeutic products has the potential to develop the market, it should not be underestimated that key for their ultimate success will closely depend on the generation of a receptive society, which needs to be educated by appropriate dissemination activities.

\section{ACKNOWLEDGMENTS}

The authors would like to thank Mrs. Anke Wixmerten for help with the editing of the text and of the figures.

\section{Reference List}


1 Pellegrini,G. et al. (1997) Long-term restoration of damaged corneal surfaces with autologous cultivated corneal epithelium. Lancet 349, 990-993

2 Tsai,R.J. et al. (2000) Reconstruction of damaged corneas by transplantation of autologous limbal epithelial cells. N. Engl. J. Med. 343, 86-93

3 Macchiarini,P. et al. (2008) Clinical transplantation of a tissue-engineered airway. Lancet 372, 2023-2030

4 Quarto,R. et al. (2001) Repair of large bone defects with the use of autologous bone marrow stromal cells. N. Engl. J. Med. 344, 385-386

5 Brun,P. et al. (2008) Characteristics of repair tissue in second-look and third-look biopsies from patients treated with engineered cartilage: relationship to symptomatology and time after implantation. Arthritis Res. Ther. 10, R132

6 Atala,A. et al. (2006) Tissue-engineered autologous bladders for patients needing cystoplasty. Lancet 367, 1241-1246

7 Lysaght,M.J. et al. (2008) Great expectations: private sector activity in tissue engineering, regenerative medicine, and stem cell therapeutics. Tissue Eng Part A 14, 305-315

8 Martin,I. et al. (2004) The role of bioreactors in tissue engineering. Trends Biotechnol. 22, 80-86

9 Kirouac,D.C. and Zandstra,P.W. (2008) The systematic production of cells for cell therapies. Cell Stem Cell 3, 369-381

10 Ratcliffe,A. and Niklason,L.E. (2002) Bioreactors and bioprocessing for tissue engineering. Ann. N. Y. Acad. Sci. 961, 210-215

11 Butler,D.L. et al. (2000) Functional tissue engineering: the role of biomechanics. $J$. Biomech. Eng 122, 570-575

12 Niklason,L.E. et al. (1999) Functional arteries grown in vitro. Science 284, 489-493

13 Hoerstrup,S.P. et al. (2001) Tissue engineering of small caliber vascular grafts. Eur. J. Cardiothorac. Surg. 20, 164-169

14 Stevens,M.M. et al. (2005) In vivo engineering of organs: the bone bioreactor. Proc. Natl. Acad. Sci. U. S. A 102, 11450-11455

15 Barbero,A. et al. (2004) Age related changes in human articular chondrocyte yield, proliferation and post-expansion chondrogenic capacity. Osteoarthritis. Cartilage. 12, 476-484

16 Braccini,A. et al. (2005) Three-dimensional perfusion culture of human bone marrow cells and generation of osteoinductive grafts. Stem Cells 23, 1066-1072

17 Scherberich,A. et al. (2007) Three-dimensional perfusion culture of human adipose tissue-derived endothelial and osteoblastic progenitors generates osteogenic constructs with intrinsic vascularization capacity. Stem Cells 25, 1823-1829

18 De Bari C. et al. (2008) A biomarker-based mathematical model to predict bone-forming potency of human synovial and periosteal mesenchymal stem cells. Arthritis Rheum. 58, 240-250

19 European Medicines Agency (2008) Guideline on human cell-based medicinal products. (http://www.emea.europa.eu/pdfs/human/cpwp/41086906enfin.pdf)

20 FDA (2008) Guidance for industry on potency tests for cellular and gene therapy products. (http://www.fda.gov/CBER/gdlns/testcellgene.htm)

21 FDA (2008) Guidance for FDA reviewers and sponsors on content and review of chemistry, manufacturing, and control (CMC) information for human gene therapy investigational new drug applications (INDs). (http://www.fda.gov/cber/gdlns/gtindcmc.htm) 
22 McAteer,H. et al. (2007) Cost-effectiveness analysis at the development phase of a potential health technology: examples based on tissue engineering of bladder and urethra. J. Tissue Eng Regen. Med. 1, 343-349

23 Mason,C. and Hoare,M. (2006) Regenerative medicine bioprocessing: the need to learn from the experience of other fields. Regen. Med. 1, 615-623

24. Pangarkar, C. and Hutmacher, D.W. (2003) Invention and business performance in the tissue-engineering industry. Tissue Eng. 9, 1313-1322

25 Mason,C. and Dunnill,P. (2008) The need for a regen industry voice. Regen. Med. 3, 621-631

26 Mason,C. and Dunnill,P. (2008) The crucial linkage required between regenerative medicine bioprocessors and clinicians. Regen. Med. 3, 435-442

Text box 1: Key opportunities and challenges for implementing bioreactors

\section{Scientifc aspects}

\section{Key Opportunity}

Sensor-based bioreactors enable unmatched ability to regulate bioprocesses to minimize process and product variability.

\section{Key Challenge}

Additional scientific insight is required to maximize the utility of bioreactor technology in providing TE grafts with consistent properties.

\section{Regulatory aspects}

\section{Key Opportunity}

Bioreactor-based processes, which include monitoring and data management systems, can offer a high level of traceability and compliance to safety guidelines.

\section{Key Challenge}

Ambiguous regulatory guidelines currently hinder the deliberate design of bioreactors which comply with specific and clear specifications.

\section{Commercial aspects}

\section{Key Opportunity}

Automated bioreactor systems may enable safe and standardized production of TE grafts, maximizing the prospective of up-scaling and cost-effectiveness in the long-term.

\section{Key Challenge}

Models for the commercialization of TE products are not well-established, resulting in uncertainties related to markets, regulatory approvals, reimbursement and overall clinical adoption. 
Table 1: Overview of Risks and Risk Mitigation for Bioreactor-based Manufacturing Systems.

\begin{tabular}{|c|c|c|}
\hline \multicolumn{2}{|c|}{ Risk } & \multirow{2}{*}{$\begin{array}{ll}\text { Risk Control/Mitigation } \\
\text { - } & \text { Closed sterilized system } \\
\text { - } & \text { Inputs sterile/clean when introduced } \\
\text { - } & \text { Contamination testing on every lot (individual patient level) } \\
\end{array}$} \\
\hline \multirow{7}{*}{ 행 } & Contamination \& Infection & \\
\hline & Toxicity/Bioburden/Pyrogens & $\begin{array}{ll}\text { - } & \text { Bioreactor and related fluid contacting surfaces selected from USP Grade } \\
\text { - } & \text { Pre-test bioreactor system with target cell type } \\
\text { - } & \text { Validate production bioburden and pyrogen levels }\end{array}$ \\
\hline & Shear Stress Damages Cells & $\begin{array}{ll}\text { - } & \text { Bioreactor system designed with benefit of fluid modeling } \\
\text { - } & \text { Validate operational sequence and maximum flow rates with target cell type } \\
\end{array}$ \\
\hline & $\begin{array}{l}\text { Compromised Cell Vitality \& } \\
\text { Performance }\end{array}$ & $\begin{array}{ll}\text { - } & \text { Biosensors for strict maintenance of culture conditions } \\
\text { - } & \text { Media and reagent sources refrigerated while on-line } \\
\text { - } & \text { Adaptive software to minimize effects of donor variability } \\
\end{array}$ \\
\hline & $\begin{array}{l}\text { Final Cell Population Insufficient for } \\
\text { Clinical Objective }\end{array}$ & $\begin{array}{ll} & \text { Establish gateway specifications in multi-step bioprocesses } \\
\text { - } & \text { Automate monitoring of cell behavior to trigger cell collection } \\
\end{array}$ \\
\hline & Sterilization Failure & - Validate according to established international standards \\
\hline & Inadequate Packaging Shelf Life & - $\quad$ Validate according to established international standards \\
\hline \multirow{5}{*}{ 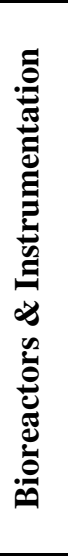 } & $\begin{array}{l}\text { Disposable Cassette Fails to Ensure } \\
\text { Consistent and Error Free Operation }\end{array}$ & $\begin{array}{ll}\text { - } & \text { Direct attachment of cell/tissue input container to minimize manual handling } \\
\text { - } & \text { Multi-layers to input container to reduce contamination risks } \\
\text { - } & \text { High level of operator visibility on process bioreactors for verification steps } \\
\text { - } & \text { Sampling ports to enable sterile withdrawal of samples (e.g. microbial testing) } \\
\text { - } & \text { Output container designed for direct transfer to clinical setting } \\
\end{array}$ \\
\hline & Bioreactor System Integrity Failure & $\begin{array}{ll} & \text { Validate connections \& methods } \\
- & 100 \% \text { leak test } \\
\text { - } & \text { Sampling program } \\
\end{array}$ \\
\hline & Sensor Reliability Issues & $\begin{array}{ll}\text { - } & \text { Sensor selection enables accurate monitoring for ex-vivo period without fouling } \\
\text { - } & \text { Operational back-up (i.e. dual monitoring \& alternate monitoring) } \\
\text { - } & \text { Validate sensor tolerance to sterilization protocol } \\
\text { - } & \text { Validate sensor shelf life when incorporated into cassette } \\
\end{array}$ \\
\hline & $\begin{array}{l}\text { Electromagnetic Interference (EMI) \& } \\
\text { Static Discharge Sensitivity }\end{array}$ & - $\quad$ Test EMI emissions and sensitivity \\
\hline & Electrical Safety Issues & - $\quad$ Meet legislative standards for safety \\
\hline \multirow{4}{*}{ 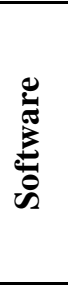 } & Code Error & $\begin{array}{ll}- & \text { Design code per standards } \\
\text { - } & \text { Rigorous code validation practices } \\
\end{array}$ \\
\hline & Software Corruption & $\begin{array}{ll}- & \text { Standard software error checking } \\
\text { - } & \text { Reload from non-volatile memory } \\
\text { - } & \text { System watch-dog } \\
\end{array}$ \\
\hline & Power Failure Recovery & $\begin{array}{ll}- & \text { Establish redundant memory } \\
\text { - } & \text { Automated recovery routines } \\
\end{array}$ \\
\hline & Operating System Compromised & - $\quad$ Code redundancy \\
\hline \multirow{3}{*}{ 岁 } & Inadvertent Misuse & $\begin{array}{ll}\text { - } & \text { Design of bioreactors and instrumentation anticipate and preclude misuse } \\
\text { - } & \text { Failsafe operation } \\
\text { - } & \text { Clear instructions for use } \\
\text { - } & \text { Field maintenance program } \\
\text { - } & \text { Remote service link-up } \\
\end{array}$ \\
\hline & Inadequate Operational Records & $\begin{array}{ll}\text { - } & \text { Process tracking and storage in non-volatile memory } \\
\text { - } & \text { Data output via computer link } \\
\text { - } & \text { Compliant with regulations on electronic records } \\
\end{array}$ \\
\hline & Unauthorized Use & - User security codes/redundancy \\
\hline
\end{tabular}




\section{Text Box 2:}

\section{SWOT Analysis for Bioreactor-based Manufacturing of Engineered Products}

- Bioreactors enable consistent implementation of bioprocesses under regulated culture conditions to maximize TE productivity

- 'Smart' bioreactor systems employing integrated sensors provide foundation for validation of TE processes for implant production

- Intelligent operation of system enables autonomous sequences to be delivered without the need for continual staff intervention

- Bioreactor control systems utilize protective measures to ensure output of process is safe and effective

- Operation of automated system and related user interface controls are easy to use within implant production facilities

- Pre-configured bioreactors and related system components enable reduced cost of routine operation, following initial set-up

- Automatic monitoring, collection and archiving of process data enhances institutional compliance with regulatory requirements

- TE production systems based on automated bioreactor technology are ideally suited to progressive scale up of process throughput

Opportunities
- Large regenerative medicine market [7] provides opportunity for

- $\frac{\text { Large regenerative medicine market [7] provides opportunity for }}{\text { TE-based product derivatives once effective implant production }}$ and delivery methods are available

- The success of a few representative models of bioreactor-based delivery of TE into the clinic will accelerate commercial \& healthcare provider interest

- Technical and administrative consistency of bioreactor-based TE supports transition and expansion from research through to clinic

- Adoption of bioreactor-based standards will enhance clinical data analysis and reinforce regulatory documentation

- Inherent consistency and quality of bioreactor output maximizes opportunity to pursue effective reimbursement for TE procedures

- Continued refinement of scientific insights on tissue engineering processes can be translated to clinic via bioreactor upgrades

- Early focus of TE in clinical therapeutics will likely focus on critical conditions that enable recovery of bioreactor R\&D costs.

\section{Weakness}

- No pre-existing market model to use as commercialization benchmark

- Operational constraints associated with bioreactor design potentially limits visualization of on-going process

- Automation requires upfront investment in capital equipment; however, follow-on costs are economical

\section{Threats}

- Cost-benefit of cell-based product may prove insufficient to compete with off-the-shelf alternative

- The use of preconfigured bioreactor assemblies and automated techniques may require the mindset of certain users to change

- Operation of system to produce cell-based implants via automated techniques potentially displaces specialized work force

- Underlying science of bioreactor-based TE may be limited by insufficient supporting data (e.g. mechanism of action)

- Innovative bioreactor technology might be constrained or delayed by mismatch with established regulatory standards

- Implementation of bioreactor-based TE strategies could be constrained or delayed by validation challenges inherent in the adoption of automated processes (e.g., lack of appropriate quality control markers) 


\section{FIGURE LEGENDS}

Figure 1. Potential model for a commercial bioreactor-based system. The translation of bioreactor-based tissue engineering into the clinic would benefit from the integration of bioreactor technology into state of the art systems which meet the safety, traceability and efficiency expected in healthcare environments. In order to eliminate the prospect of cross contamination, the bioreactor and associated media management would be ideally isolated on a patient-specific level through the use of disposable bioreactor 'cartridges' or 'cassettes'. Automated operational control over the cassette would occur via a space-efficient, multichannel (i.e. multi-patient) instrument that operates under password-protected security access. Initiation and monitoring of the process underway for each patient would occur via an intuitive graphical interface that provides an accurate and timely indication of status operation, along with secure data archiving. As the process advances within the cassette, biosensors associated with the bioreactors and media management system provide feedback on tissue culture conditions and enable the control system to maintain critical process parameters. Upon completion of the process, the cassette would enable the removal of an output cartridge that inherently protects the internal vial during the final stages of transport to the surgical center for implantation. Automated cell processing systems have been developed by several groups such as Aastrom Biosciences Inc., (http://www.aastrom.com) and Tissue Genesis Inc. (http://www.tissuegenesis.com). The example illustrated is under development by Octane Biotech Inc. (http://www.octaneco.com/biotech) with the essential goal of enabling full GMP processing on a miniature and cost-effective scale.

Figure 2. A proposed roadmap for translating bioreactor-based engineered products into the clinic. Highlighted are the most critical scientific, regulatory, and commercial challenges that will need to be addressed along the path irrespective of the particular tissue engineering application. 
Once embarking into the development of a bioreactor-based tissue engineering system, and targeting a specific clinical application with clear goals in terms of projected clinical outcome, core biological criteria associated with the engineered product, the relevant bioprocesses, and basic regulatory guidelines must be established and verified. Planning and conducting sound pre-clinical studies will allow to further assessing and validating the criteria that had been previously established in the core biology platform, most likely leading to refinements and optimization of bioprocesses as well as to a better understanding of the underlying biology. Interactions with industrial partners will facilitate the transition between platforms, through the evolution and industrialization of research based technologies as well as by defining the commercial potential for the product. In the final stage of the roadmap, which clearly is the most challenging and critical, the translation to the clinic platform will engage clinical, regulatory and reimbursement strategies [26]. 